\title{
Prevalence and prognostic significance of microsatellite alterations in young patients with bladder cancer
}

\author{
Mario Migaldi ${ }^{1}$, Giuliana Sartori ${ }^{1}$, Giulio Rossi ${ }^{1}$, Lorella Garagnani ${ }^{1}$, Beatrice Faraglia ${ }^{2}$, \\ Carmela De Gaetani ${ }^{1}$, Achille Cittadini ${ }^{2}$, Gian P Trentini ${ }^{1}$ and Alessandro Sgambato ${ }^{2}$ \\ ${ }^{1}$ Dipartimento Misto di Anatomia Patologica e di Medicina Legale, Sezione di Anatomia Patologica, \\ University of Modena and Reggio Emilia, Modena, Italy and ${ }^{2}$ Centro di Ricerche Oncologiche 'Giovanni \\ XXIII', Istituto di Patologia Generale, Catholic University, Rome, Italy
}

\begin{abstract}
Mutations in microsatellite sequences are a hallmark of neoplastic transformation and have been reported in the majority of human cancers. Conflicting results have been reported on the role of microsatellite alterations in bladder tumorigenesis and it has been suggested that they might be mainly involved in the development of bladder cancers in young patients. In this study, DNA was extracted from laser-microdissected samples of $\mathbf{5 1}$ superficial papillary bladder urothelial carcinomas arising in young patients and was analyzed for the status of 19 microsatellite loci previously reported to be associated with bladder tumorigenesis. The occurrence and the pattern of microsatellite alterations, in form of loss or length variation, was evaluated and correlated with other clinicopathologic and molecular markers. The prognostic significance of these alterations was also evaluated. Loss of heterozygosity at one or more loci was detected in all 51 tumors analyzed. Length variation in at least one locus was observed in $48(94 \%)$ of the cases. The microsatellite that was more frequently altered was D11S488 $(69 \%)$, followed by D9S162 $(61 \%)$, D3S3050 $(55 \%)$, D3S1300 $(51 \%)$ and D4S243 $(51 \%)$, all the remaining being altered in less than $50 \%$ of cases. The occurrence of microsatellite alterations was not associated with tumor grade nor with tumor stage, the expression of p53, cyclin D1 or the cyclin-dependent kinase-inhibitor p27 $7^{\mathrm{kip} 1}$ while it was significantly more frequent in tumors with increased expression of the proliferation marker MIB-1 $(P=0.003)$. The occurrence of alterations at the analyzed loci was associated with a reduced risk of tumor recurrence $(P=0.04$ by log-rank test) and disease progression $(P=0.02)$ in a univariate analysis. These findings demonstrate that microsatellite alterations are frequent and early events and might have a prognostic significance in bladder cancers arising at young age.

Modern Pathology (2005) 18, 1176-1186. doi:10.1038/modpathol.3800399; published online 22 April 2005
\end{abstract}

Keywords: bladder cancer; microsatellite alterations; young; cell cycle; prognosis

The bladder urothelial carcinoma represents the fifth most common cancer following lung, breast, colorectal and prostate malignancies ${ }^{1}$ and accounts for about $2.1 \%$ of all cancer-related deaths. ${ }^{1}$ It affects both genders with a male to female ratio of $4: 1$ and the highest rate of incidence in the sixth decade of life. Thus, it is uncommon (about 1-1.5\%) before 40 years of age ${ }^{2}$ and it is still unclear whether cancers

Correspondence: Dr A Sgambato, MD, PhD, Istituto di Patologia Generale, Centro di Ricerche Oncologiche 'Giovanni XXIII', Università Cattolica del Sacro Cuore, Largo Francesco Vito 1, 00168 Rome, Italy.

E-mail: asgambato@rm.unicatt.it

Received 2 November 2004; revised 12 January 2005; accepted 13 January 2005; published online 22 April 2005 arising in younger patients proceed through the same molecular pathways than their older counterparts.

Microsatellites are short (1-10 base pairs) repetitive sequences that occur primarily in noncoding regions of DNA. ${ }^{3}$ The number of microsatellite repeat units located at a given locus is genetically determined and has been very useful as marker for genetic mapping. ${ }^{4}$ However, because of their repetitive structure, microsatellites are susceptible to slippage errors during DNA replication, resulting in insertion or deletion mutations.

Frequent occurrence of loss and length alteration of microsatellites has been described for bladder urothelial carcinomas. ${ }^{5}$ Loss of microsatellites has been used as a marker of loss of heterozygosity (LOH) and has been shown to involve several loci 
throughout the genome ${ }^{6,7}$ with chromosome 9 being the most frequently altered in both superficial (pTa-T1) and muscle-invasive bladder cancers. ${ }^{8}$ The accumulation of mutations in microsatellite sequences throughout the genome is known as microsatellite instability. Microsatellite instability is a form of genomic instability characterized by variations in the length of microsatellite repeats and is a hallmark of neoplastic transformation. Two different types of microsatellite instability have been described in human cancers. The 'classical' microsatellite instability is typical of hereditary nonpolyposis colorectal cancers (HNPCC) and is associated with germline mutations in genes involved in DNA mismatch repair mechanisms. ${ }^{9}$ Conflicting results have been reported on the frequency of microsatellite instability in bladder cancers with values ranging from 0 to $100 \% \cdot{ }^{10-17}$ More recently, a second variety of instability has been reported that appears to be not related to DNA mismatch repair deficiency and is best seen at tetranucleotide repeats. ${ }^{11}$ This form of instability has been termed EMAST (elevated microsatellite alterations at selected tetranucleotides) and appears to occur with high frequency (approximately 40\%) in bladder cancer. ${ }^{11,18}$ Limited data have been reported so far on the occurrence of microsatellite alterations in bladder urothelial carcinomas arising in young patients. ${ }^{17}$

Several molecular markers have been identified in the last years, which play an important role in the process of tumor development. Some of the most recent and promising include cell proliferationrelated markers, which have been also associated with the biological and clinical behavior of bladder cancers. ${ }^{19,20}$ In fact, abnormalities in the expression and/or activity of various cell cycle control proteins frequently occur during the multistage process of carcinogenesis and play a critical role in both the process of tumor progression and in maintenance of the malignant phenotype. ${ }^{21}$ The tight regulation of cell cycle progression, however, is essential to ensure the proper duplication of DNA. Thus, deregulation of normal cell cycle can induce genome instability and promote the accumulation of mutations throughout the genome, including the microsatellite regions. No study have been reported that analyzes the relationship between abnormalities in the expression of cell cycle-related proteins and the occurrence of microsatellite alterations in bladder cancer.

In this study, we have analyzed a large number of microsatellite loci to detect microsatellite alterations in a series of homogeneously treated bladder cancers arising in young patients and compared the results obtained with the clinical evolution of tumors and with the expression of several cell proliferation markers. We found that microsatellite alterations are a frequent event and might have prognostic significance in bladder urothelial carcinomas arising in young patients. The implications of these findings are discussed.

\section{Materials and methods}

\section{Patients Selection}

The 2.077 cases (first diagnosis) of bladder carcinomas recorded between 1991 and 2001 at the Department of Pathologic Anatomy of the University of Modena and Reggio Emilia were retrospectively searched for 'young' patients. The 45 years of age was selected as the threshold in discriminating between young and elderly group of patients with bladder urothelial carcinoma based on the recommendations of the National Cancer Data Base. ${ }^{22}$

A total of 58 cases of bladder urothelial carcinomas arising in patients aged 45 years or younger, for which detailed clinicopathologic data were available, were identified and retrieved. They were blindly reviewed by two of the authors independently (GR and GPT). Two cases with discrepant diagnosis about tumor grade were re-evaluated jointly at a multiheaded microscope and agreement was reached. Histologic grade and stage were assessed according to WHO classification ${ }^{23}$ and $\mathrm{TNM}^{24}$ staging system, respectively. Retrieved tumors included 51 superficial (pTa-T1), two invasive (pT2-4) and six in situ carcinomas. In order to analyze a homogeneous series of tumors, only patients with superficial tumors were used for this study. They included 34 $(67 \%)$ men and $17(33 \%)$ women with a male to female ratio of $2: 1$ and a mean age at diagnosis of 38 years (range 20-45 years). The mean follow-up was 65 months with a range of 2 to 156 months. Tumor stage was pTa in 36 cases $(71 \%)$ and pT1 in the remaining 15 cases (29\%). In all, 13 (25\%) cases were classified as well (G1), 29 (57\%) as moderately (G2) and $9(18 \%)$ as poorly (G3) differentiated tumors. The series only included papillary tumors.

All patients underwent transurethral resection with curative intent, and none of them had received any therapy before surgery. Patients diagnosed with pT1 tumors were treated by intravescical Bacillus Calmette-Guerin (BCG) instillations after surgery and all patients received intravescical BCG in case of recurrence. Follow-up was performed by cystoscopy every 3 months for the first year, every 4 months in the second and third year, every 6 months for the fourth and fifth year, and annually thereafter if there was no recurrence. Recurrence was defined as the occurrence of a positive cystoscopy coupled to a biopsy-proved lesion. Progression was defined as the development of muscle-invasive or more advanced stage carcinoma, distant metastasis, or death from bladder cancer. The selection did not require approval by an Institutional Review Board because the samples were coded and the names of the patients were not revealed.

\section{Microsatellite Analysis}

We selected 19 microsatellite markers (Table 1) based on their informativity and frequency of 
Table 1 Microsatellite loci, primer sequences and type of repetitive sequence analyzed in a series of bladder cancers arising at young age

\begin{tabular}{|c|c|c|}
\hline Locus (chromosomal arm) & Primer sequences & Type of repetition \\
\hline D1S2883 (1p) & $\begin{array}{l}\text { AAATCTGGTCTTCTGTTTTCACTAT } \\
\text { TTCCAAATGTTGACTCTGC }\end{array}$ & Dinucleotide \\
\hline D3S3050 (3p) & $\begin{array}{l}\text { TGGTGGTATGCATTTGTCAG } \\
\text { ATTCCCTGACTTCAAGTGCA }\end{array}$ & Tetranucleotide \\
\hline D3S1300 (3p) & $\begin{array}{l}\text { AGCTCACATTCTAGTCAGCCT } \\
\text { GCCAATTCCCCAGATG }\end{array}$ & Dinucleotide \\
\hline D3S2418 (3q) & $\begin{array}{l}\text { GTACATTCTGCACACGTACCC } \\
\text { AGGACTGTAGGATTCCAAAGG }\end{array}$ & Trinucleotide \\
\hline D4S243 (MH34) (4p) & $\begin{array}{l}\text { TCAGTCTCTCTTTCTCCTTGCA } \\
\text { TAGGAGCCTGTGGTCCTGTT }\end{array}$ & Tetranucleotide \\
\hline D4S243 (SHGC) (4p) & $\begin{array}{l}\text { AATCCCTTTTCTACCTTTCTATCAC } \\
\text { GAGAGGAGAGATAAAAGATGTAAATG }\end{array}$ & Tetranucleotide \\
\hline D5S346 (5q) & $\begin{array}{l}\text { ACTCACTCTAGTGATAAATCGGG } \\
\text { AGCAGATAAGACAGTATTACTAGTT }\end{array}$ & Dinucleotide \\
\hline D9S54 (9p) & $\begin{array}{l}\text { GAAAGTCCAGAACTAAGTAG } \\
\text { TGTGGATAGGTATATATAGC }\end{array}$ & Dinucleotide \\
\hline D9S747 (9q) & $\begin{array}{l}\text { GCCATTATTGACTCTGGAAAAGAC } \\
\text { CAGGCTCTCAAAATATGAACAAAAT }\end{array}$ & Tetranucleotide \\
\hline D9S162 (9p) & $\begin{array}{l}\text { GCAATGACCAGTTAAGGTTC } \\
\text { AATTCCCACAACAAATCTCC }\end{array}$ & Dinucleotide \\
\hline IFNA (9p) & $\begin{array}{l}\text { TGCGCGTTAAGTTAATTGGTT } \\
\text { GTAAGGTGGAAACCCCCACT }\end{array}$ & Dinucleotide \\
\hline D9S171 (9p) & $\begin{array}{l}\text { AGCTAAGTGAACCTCATCTCTGTCT } \\
\text { ACCCTAGCACTGATGGTATAGTCT }\end{array}$ & Dinucleotide \\
\hline D9S164 (9q) & $\begin{array}{l}\text { CTATGATCGTGCCACTGCAC } \\
\text { GTTTGCCTGGGGATTGATTT }\end{array}$ & Dinucleotide \\
\hline D11S488 (11q) & $\begin{array}{l}\text { AGGCAATAGAGACCCTGTG } \\
\text { GATGATGAATTGTTACTGAGAG }\end{array}$ & Tetranucleotide \\
\hline D13S802 (13q) & $\begin{array}{l}\text { CACAGTGAGACTCTATCTCAAAAA } \\
\text { TCAGACTGGCTTAGACTGTGG }\end{array}$ & Tetranucleotide \\
\hline D16S310 (16q) & $\begin{array}{l}\text { GGGCAACAAGGAGAGACTCT } \\
\text { AAAAAAGGACCTGCCTTTATCC }\end{array}$ & Tetranucleotide \\
\hline D16S476 (16q) & $\begin{array}{l}\text { TTGCACTCCACTCTGGGCA } \\
\text { TTGCCTTGGCTTTCTGTTGG }\end{array}$ & Pentanucleotide \\
\hline D17S654 (17p) & $\begin{array}{l}\text { GACCTAGGCCATGTTCACAGCC } \\
\text { GACATCCATTGGCACCACCCCAA }\end{array}$ & Dinucleotide \\
\hline ТР53 (17p) & $\begin{array}{l}\text { AGGGATACTATTCAGCCCGAGGTG } \\
\text { ACTGCCACTCCTTGCCCCATT }\end{array}$ & Dinucleotide \\
\hline
\end{tabular}

alteration in bladder cancer. These 19 markers are located on nine chromosomes, including 11 chromosomal arms, and have been previously reported to be associated with human bladder tumorigenesis. ${ }^{10-17}$ Genomic DNA was prepared from $5 \mu \mathrm{m}$-thick paraffin-embedded tissue sections according to the standard protocols. In brief, histological sections were deparaffinized by a 1-h incubation at $65^{\circ} \mathrm{C}$ followed by incubation in xylene. They were then rehydrated in a series of decreasing ethanol concentrations and stained with hematoxylin/eosin. By laser microdissection, approximately $0.5 \mathrm{~cm}^{2}$ of tumor and normal tissues were prepared from each section, respectively.

Microdissection of clear-cut cancer cells was performed under direct observation with an inverted microscope using a laser capture microdissection apparatus (Olympus IX70, Laser Scissors Pro 300, Olympus Italia, Segrate, Milan, Italy) controlled by a joystick-operated electronic manipulator. The microdissected samples were transferred to an eppendorf tube containing digestion buffer $(2 \mathrm{mg} / \mathrm{ml}$ proteinase $\mathrm{K}$ in $10 \mathrm{mM}$ Tris/HCl (pH8.0), $2.5 \mathrm{mM} \mathrm{MgCl}_{2}, 0.5 \%$
Tween 20). The tubes were then incubated for $24 \mathrm{~h}$ at $37^{\circ} \mathrm{C}$, followed by $10 \mathrm{~min}$ at $95^{\circ} \mathrm{C}$ to eliminate any remaining proteinase K activity. PCR was performed in $10 \mu \mathrm{l}$ reaction mixture containing 25 pmol each of primers, $1 \mu \mathrm{l}$ of extracted DNA from at least 100 cells, $200 \mu \mathrm{M}$ each of deoxynucleoside triphosphate, $2 \mathrm{mM}$ magnesium chloride, $1 \mu \mathrm{l}$ of commercial PCR buffer (Applied Biosystem, Applera Italy, Monza, Italy) and 1 unit of AmpliTaq Gold polymerase (Applied Biosystem). The PCR conditions were as follows: after an initial 2-min denaturation step at $95^{\circ} \mathrm{C}, 40$ amplification cycles were performed, each consisting of denaturation $\left(30 \mathrm{~s}\right.$ at $\left.95^{\circ} \mathrm{C}\right)$, annealing (30 $\mathrm{s}$ at $55-60^{\circ} \mathrm{C}$, depending on the primers), and elongation $\left(30 \mathrm{~s}\right.$ at $72^{\circ} \mathrm{C}$ ) steps followed by a final step at $72^{\circ} \mathrm{C}$ for $10 \mathrm{~min}$. The amplified PCR products were then run on a $4 \%$ denaturing polyacrylamide gel for the ABI-Prism 310 automatic sequencer (Applied Biosystem) with a labelled marker (TAMRA 500) as an internal size standard. The run was performed in accordance with the supplier's protocol. Nineteen primer sequences (MWG-Biotech, Florence, Italy) flanking 19 microsatellite repeat 
polymorphisms located at 18 chromosomal regions of nine different chromosomes were used, as follows: D1S2883, D3S3050, D3S1300, D3S2418, D4S243 (MH34), D4S243 (SHGC), D5S346, D9S54, D9S747, D9S162, IFNA, D9S171, D9S164, D11S488, D13S802, D16S310, D16S476, D17S654, and TP53 (Table 1). The data were analyzed with a Genescan 3.0 software (Applied Biosystem). Microsatellite analysis was performed by comparing the positions of the bands corresponding to the tumor and the normal DNAs according to the manufacturer's manual. Briefly, peak height of each microsatellite locus for independent injections of each normal and each tumor sample was obtained. Length variation was determined when the electropherogram showed the presence of novel peaks, with an evident shift, in the tumor DNA, that were not present in normal DNA (Figure 1). LOH was also detected and was defined as the loss of a wild-type allele (peak) in tumor compared with normal DNA (Figure 1). The mathematic model of $\mathrm{LOH}$ determination used is the following:

height of normal allele two/height of normal allele one height of tumor allele two/height of tumor allele one

An $\mathrm{LOH}$ value $\leq 0.5$ indicated that the tumor sample showed significant loss of the longer allele whereas an $\mathrm{LOH}$ value $\geq 1.5$ indicated a significant loss of the shorter allele. Results were confirmed in all cases in duplicate experiments using independently extracted DNA samples.

\section{Immunohistochemical Analyses}

All immunohistochemical analyses were performed on routinely processed, formalin-fixed, paraffinembedded tissues, employing an avidin-biotin complex immunoperoxidase technique (Vectastain ABC kit; Vector Laboratories, Burlingame, CA, USA) as previously described. ${ }^{20,25}$ Briefly, $5-\mu \mathrm{m}$ sections were cut from representative block and subsequently dewaxed, rehydrated and then microwave pretreated $(10 \mathrm{~min}$ at $750 \mathrm{~W}$ in $10 \mathrm{mM}$ citrate buffer at $\mathrm{pH}$ 6.0). Sections were then incubated with $0.3 \%$ hydrogen peroxide in methanol for $30 \mathrm{~min}$ to block endogenous peroxidase. After blocking for $1 \mathrm{hr}$ at room temperature, the primary antibodies were applied overnight at $4^{\circ} \mathrm{C}$ in a high-humidity chamber. Binding was visualized using the Vectastain diaminobenzidine kit (Vector Laboratories) and counterstaining was performed with $1 \%$ modified Meyer's hematoxylin. Antibodies against the following proteins were used: cyclin D1 (monoclonal; Novocastra, Newcastle upon Tyne, UK; 1:10 dilution), Ki67 (monoclonal, clone MIB-1; Dako, Glostrup, Denmark; 1:100 dilution), p53 (monoclonal, clone D07; Dako; 1:750 dilution) and p27 ${ }^{\text {Kip1 }}$ (polyclonal; Santa Cruz Biotechnology, Santa Cruz, CA, USA; 1:100 dilution). Controls for specificity of staining were performed by immuno- staining duplicate sections in the absence of the primary antibody. Sections of breast carcinomas known to be positive for each of the markers analyzed, respectively, served as a positive control. Positive and negative control slides were included within each batch of slides. For all markers, immunostained nuclei were considered positive when they showed a distinct brown color in the absence of background staining. The fraction of positive-stained nuclei was scored by examining at least 10 random high-power field $(\times 400)$ for each samples and the percentage of cells with positive reaction was calculated semiautomatically by means of a computer-assisted cellular image analyzer on a total of at least 1000 tumor nuclei per case. All scoring and interpretations of the results were performed by two of the authors independently (AS and MM) without the knowledge of clinical outcome or other clinicopathologic variables. To assess interobserver variations, the results of the two measurements were compared by paired $t$-test and no statistical differences were found (data not shown). The few cases with discrepant scoring (4 cases) were re-evaluated jointly on a second occasion, and an agreement was reached.

\section{Statistical Analysis}

The association between microsatellite alterations and other molecular and clinicopathological parameters were calculated using contingency table methods and tested for significance using the Fisher's exact test. The Bonferroni correction was used to correct for multiple comparisons. Survival curves were calculated using the Kaplan-Meier method and the log-rank test was used for the analysis. Univariate and multivariate relative risks were calculated using Cox proportional hazards regression. Time to recurrence was calculated from operation to the first documented recurrence. Time to disease progression was calculated from operation to the first documented clinical progression, namely muscle invasion, with or without invasion into surrounding tissues, lymph node or distant metastases, or death from tumor. In the absence of recurrence/progression, follow-up time was calculated from operation to the date of the last documented follow-up. For grade and stage, the G1 grade and the pTa stage, were used as baseline, respectively. All calculations were performed by the SPSS rel. 8.00 (Statistical Package for Social Science) and the results were considered statistically significant when the $P$-value was $\leq 0.05$

\section{Results}

The clinicopathological characteristics and results of the microsatellite analysis are summarized in Tables 2 and 3. 

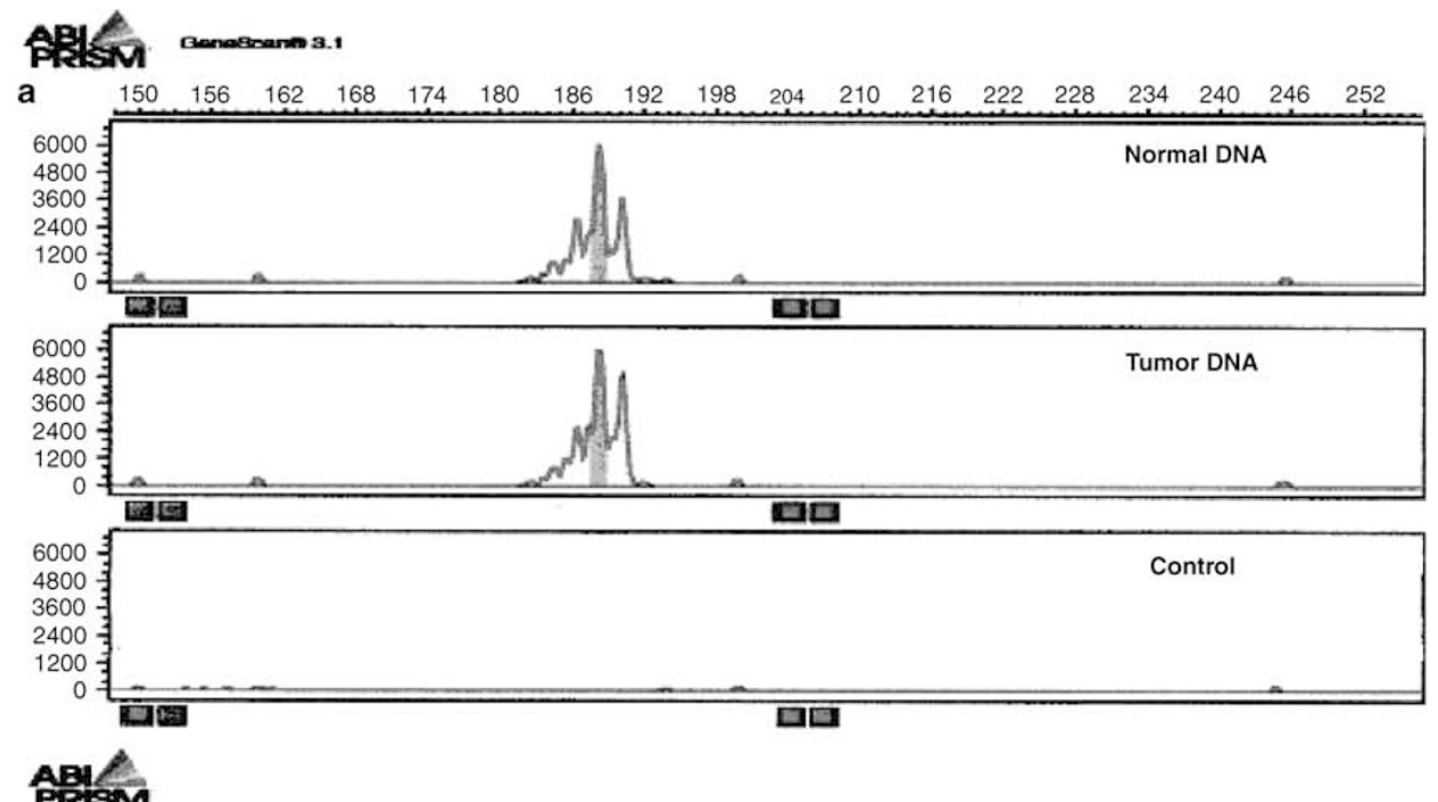

$\begin{array}{lllllllllllllllllllll}\text { b } & 80 & 84 & 88 & 92 & 96 & 100 & 104 & 108 & 112 & 116 & 120 & 124 & 128 & 132 & 136 & 140 & 144 & 148 & 152 & 156\end{array}$
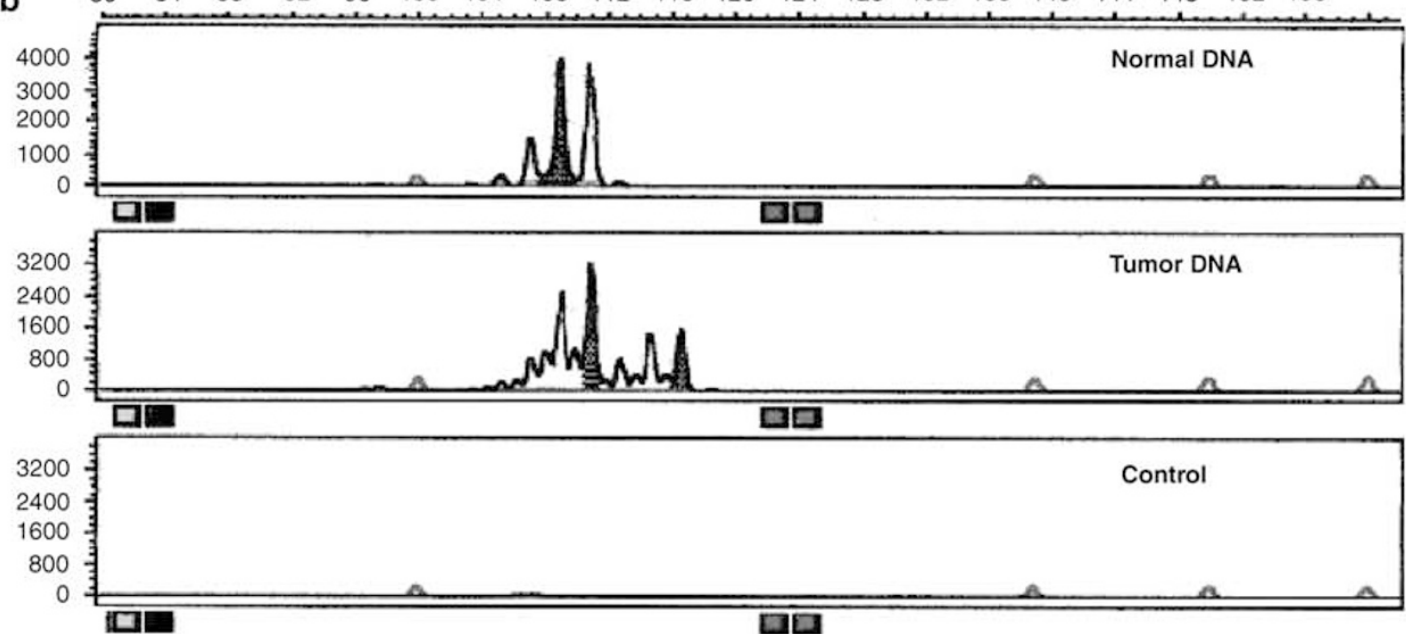

ARISM

C $\quad \begin{array}{llllllllllllllllllll}222 & 228 & 294 & 240 & 246 & 252 & 258 & 264 & 270 & 276 & 282 & 288 & 294 & 300 & 306 & 312 & 318 & 324 & 330 & 336\end{array}$

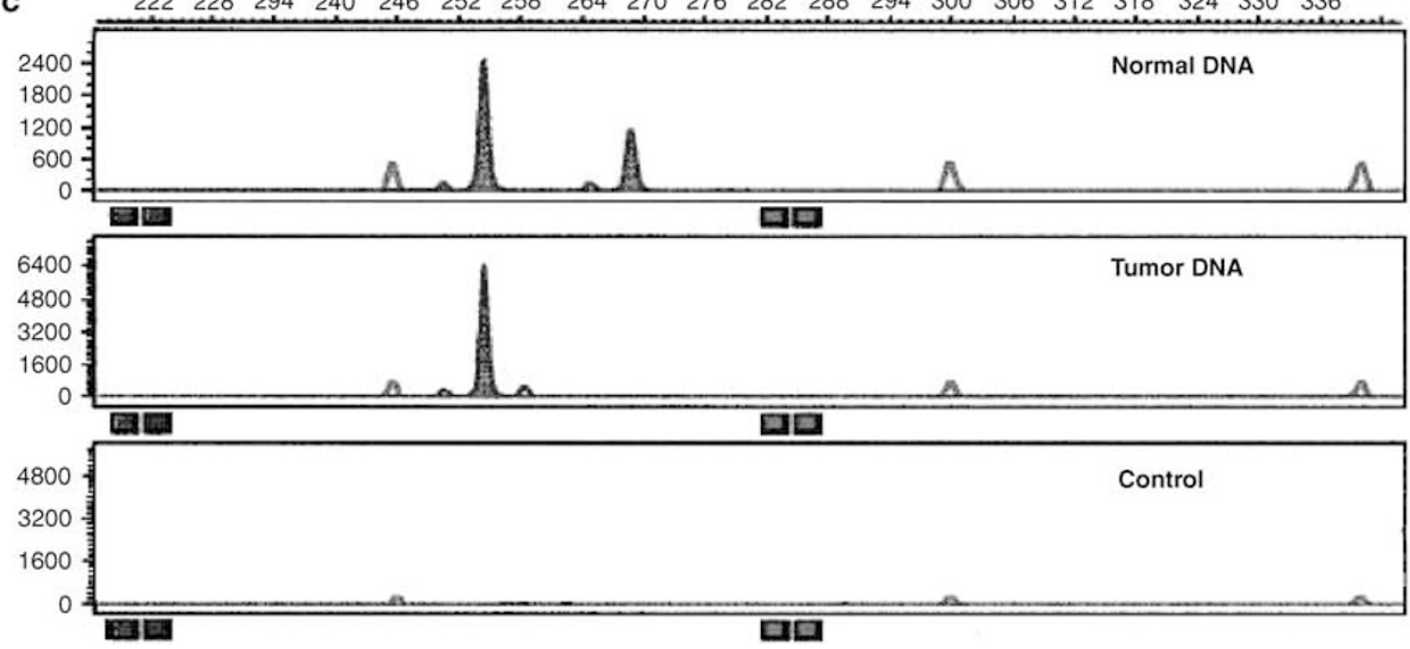


$\mathrm{LOH}$ at one or more loci was detected in all 51 tumors analyzed with a mean of 3.2 deleted microsatellites for each case (range 1-8; median 3). Length variation was observed in 48 (94\%) cases with a mean of 4.9 altered microsatellites for each case (range 0-11; median 5). Overall, alterations of at least one of the selected loci, in the form of deletion or alteration of peak(s), was observed in all tumors with a mean of $8(42 \%)$ altered microsatellites of the 19 examined for each tumor (range 1-16; median 8) (Table 2).

Based on these results, we used the median values to classify tumors as displaying a high or a low frequency of microsatellite alterations, as previously suggested. ${ }^{26}$ Thus, tumors were classified as displaying high frequency of microsatellite alterations if they showed more than five shifts and/or more than three $\mathrm{LOH}$ in the loci analyzed. A total of 31 tumors $(61 \%)$ fell in this category (10 tumors with more than five shifts, 11 with more than three LOH and 10 displaying both types of alterations) with the remaining $20(39 \%)$ being classified as displaying low frequency of microsatellite alterations. As shown in Table 3, no relationship was observed between microsatellite alterations and patients gender in our series of bladder urothelial carcinomas arising at young age. No relationship was also

Table 2 Status of 19 genetic loci in a series of 51 superficial papillary bladder urothelial carcinomas arising in young patients

\begin{tabular}{lcrc}
\hline Locus & $\begin{array}{c}\text { Length variation } \\
\mathrm{n}(\%)\end{array}$ & $\begin{array}{c}\text { LOH } \\
\mathrm{n}(\%)\end{array}$ & $\begin{array}{c}\text { Unaltered } \\
\mathrm{n}(\%)\end{array}$ \\
\hline D1S2883 & $16(31)$ & $8(16)$ & $27(53)$ \\
D3S3050 & $20(39)$ & $8(16)$ & $23(45)$ \\
D3S1300 & $14(27)$ & $12(24)$ & $25(49)$ \\
D3S2418 & $6(12)$ & $9(18)$ & $36(71)$ \\
D4S243 (SHGC) & $19(37)$ & $7(14)$ & $25(49)$ \\
D4S243 (MH34) & $16(31)$ & $9(18)$ & $26(51)$ \\
D5S346 & $9(18)$ & $11(22)$ & $31(61)$ \\
D9S54 & $11(21)$ & $3(6)$ & $37(73)$ \\
D9S747 & $7(14)$ & $11(21)$ & $33(65)$ \\
D9S162 & $22(43)$ & $9(18)$ & $20(39)$ \\
IFNA & $1(2)$ & $5(10)$ & $45(88)$ \\
D9S171 & $13(25)$ & $11(22)$ & $27(53)$ \\
D9S164 & $14(27)$ & $8(16)$ & $29(57)$ \\
D11S488 & $23(45)$ & $12(24)$ & $16(31)$ \\
D13S802 & $16(31)$ & $9(18)$ & $26(51)$ \\
D16S310 & $13(25)$ & $10(20)$ & $28(55)$ \\
D16S476 & $9(18)$ & $6(12)$ & $36(71)$ \\
D17S654 & $10(20)$ & $6(12)$ & $35(69)$ \\
TP53 & $9(18)$ & $4(8)$ & $38(75)$ \\
& & & \\
\hline
\end{tabular}

Length variation: indicates the occurrence of shifts in the DNA peaks; LOH: indicates the loss of a wild-type allele (peak); Unaltered: indicates the absence of alterations between the corresponding tumor and normal DNAs. observed between the occurrence of microsatellite alterations and tumor stage and grade.

The expression of several cell cycle-related markers, such as MIB-1, cyclin D1, p53 and $\mathrm{p} 27^{\mathrm{Kip} 1}$, was also evaluated by immunostaining in the series of young bladder urothelial carcinomas analyzed in this study. As previously reported,

Table 3 Clinicopathological parameters and microsatellite alterations in 51 superficial papillary bladder urothelial carcinomas arising in young patients $(\%)$

\begin{tabular}{|c|c|c|c|c|}
\hline & Total & $\begin{array}{c}M S I \\
(\mathrm{n}=20)\end{array}$ & $\begin{array}{c}L O H^{\mathrm{a}} \\
(\mathrm{n}=21)\end{array}$ & $\begin{array}{c}\text { Low } M A \\
(\mathrm{n}=20)\end{array}$ \\
\hline \multicolumn{5}{|l|}{ Gender } \\
\hline Male & $34(67)$ & 12 (35) & $14(41)$ & $14(41)$ \\
\hline Female & $17(33)$ & $8(47)$ & $7(41)$ & $6(35)$ \\
\hline \multicolumn{5}{|c|}{ Tumor stage } \\
\hline pTa & $36(71)$ & $15(42)$ & $16(44)$ & $12(33)$ \\
\hline pT1 & $15(29)$ & $5(33)$ & $5(33)$ & $8(53)$ \\
\hline \multicolumn{5}{|c|}{ Tumor grade } \\
\hline G1 & $13(25)$ & $7(54)$ & $4(31)$ & $4(31)$ \\
\hline G2 & $29(57)$ & $13(45)$ & $16(55)$ & $8(28)$ \\
\hline G3 & $9(18)$ & $0(0)$ & 1 (11) & $8(88)$ \\
\hline \multicolumn{5}{|l|}{ Cyclin D1 } \\
\hline High & $30(59)$ & $12(40)$ & $11(37)$ & $10(33)$ \\
\hline Low & $21(41)$ & $8(38)$ & $10(48)$ & $10(48)$ \\
\hline \multicolumn{5}{|l|}{ p27Kip1 } \\
\hline High & $18(35)$ & $5(28)$ & $8(44)$ & 7 (39) \\
\hline Low & $33(65)$ & $15(45)$ & $13(39)$ & $13(39)$ \\
\hline \multicolumn{5}{|l|}{ MIB1 } \\
\hline High & $22(43)$ & $6(27)$ & $6(27)$ & $14(64)^{*}$ \\
\hline Low & $29(57)$ & $14(48)$ & $15(52)$ & $6(21)$ \\
\hline \multicolumn{5}{|l|}{ p53 } \\
\hline High & $28(55)$ & 13 (46) & $7(25)$ & $13(46)$ \\
\hline Low & $23(45)$ & $7(30)$ & $14(61)$ & $7(30)$ \\
\hline \multicolumn{5}{|c|}{ Recurrence } \\
\hline Yes & $21(41)$ & $7(33)$ & $7(33)$ & $12(57)^{*}$ \\
\hline No & $30(59)$ & $13(43)$ & $14(47)$ & $8(27)$ \\
\hline \multicolumn{5}{|c|}{ Progression } \\
\hline Yes & $8(16)$ & $2(25)$ & $2(25)$ & $6(75)^{*}$ \\
\hline No & $43(84)$ & $18(42)$ & $19(44)$ & 14 (33) \\
\hline
\end{tabular}

MSI: indicates the occurrence of shifts in the DNA peak of more than five loci; $\mathrm{LOH}$ : indicates the occurrence of deletions in more than three loci; Low MA: include cases displaying neither MSI nor LOH. ${ }^{\mathrm{a}}$ Some cases displayed both MSI and LOH.

* Statistical analyses were performed by the Fisher's exact test considering cases with Low MA vs the remaining ones for all variables. $P \leq 0.05$ was considered significant. None of the variables, but MIB-1 expression $(P=0.003)$, recurrence $(P=0.04)$ and disease progression $(P=0.04)$ reached significance.

Figure 1 Electropherograms of microsatellite analysis using the automated single-capillary ABI PRISM sequencer model 310 Genetic Analyzer. Control = PCR control without DNA. Small peaks correspond to DNA molecular weight standard. The electropherograms of normal and tumor DNA are shown. (a) No differences are evident between them; (b) an additional peak is observed in tumor DNA; (c) loss of an allele is observed in tumor compared to normal DNA. 
immunopositivity was defined as high if nuclear staining was detectable in more than $25 \%$ of cells for cyclin D1, p53 and p2 $7^{\mathrm{Kip} 1}$ and in more than $15 \%$ of cells for MIB-1. ${ }^{26,27}$ High expression of MIB-1 was detected in eight $(26 \%)$ cases displaying high frequency of microsatellite alterations and in 14 $(70 \%)$ with low frequency of microsatellite alterations and this difference was significant $(P=0.003)$. On the other hand, expression of cyclin D1, p53 and of the CDK-inhibitor p27 ${ }^{\mathrm{Kip} 1}$ was not significantly different in the two groups of tumors (Table 3). No other significant relationships were observed considering the occurrence of length variations or $\mathrm{LOH}$ separately.

In all, 21 patients $(41 \%)$ recurred during the period of follow-up with eight of them (38\%) progressing to muscle-invasive $(\mathrm{pT} 2+)$ disease. The recurrent patients included nine of the 36 $(25 \%)$ pTa and 12 of the $15(80 \%)$ pT1 tumors and this difference was significant $(P<0.001)$. In a univariate analysis, high tumor stage was significantly associated with a shorter disease-free survival in our series of patients $(P=0.0004$ by log-rank test) (Figure 2a). Recurrence also occurred in two (15\%) of the $13 \mathrm{G} 1$, in $13(45 \%)$ of the $29 \mathrm{G} 2$ and in six (67\%) of the nine G3 tumors. These differences were significant (0.01 at $\chi^{2}$ test for trend) and in a univariate analysis high tumor grade was significantly associated with a shorter disease-free survival in our series of patients $(P=0.04$ by log-rank test $)$ (Figure 2b). None of the available molecular markers displayed any relationship with tumor recurrence in our group of patients. However, Kaplan-Meier curves showed that low cyclin D1 expression $(P=0.037)$ and high MIB-1 index $(P=0.04)$, but not reduced expression of p27 ${ }^{\text {Kip } 1}$ or p53 overexpression, were associated with an increased risk of recurrence (data not shown).
Nine $(29 \%)$ of the 31 cases displaying high frequency of microsatellite alterations and 12 $(60 \%)$ of the remaining ones recurred in our series of young bladder urothelial carcinomas during the period of follow-up and this difference was significant $(P=0.04)$ (Table 3$)$. The Kaplan-Meier curves of disease-free survival displayed a significant separation when tumors with high frequency of microsatellite alterations were stratified vs the remaining ones demonstrating that the occurrence of alterations at the selected loci was associated with a reduced risk of recurrence in a univariate analysis $(P=0.039$ by log-rank test) (Figure 2c).

In a multivariate analysis, performed building a Cox proportional hazards model that included all of the variables that were significant at the univariate analysis (ie, tumor stage and grade, cyclin D1 and MIB1 expression and frequency of microsatellite alterations), only higher tumor stage $(P=0.001$; relative risk $=1.7 ; 95 \%$ confidence interval: $1.36-$ 12.63) confirmed to be and independent predictor of shorter disease-free survival while frequency of microsatellite alterations $(P=0.08$; relative risk $=$ 1.08; 95\% confidence interval: $0.97-8.96)$ and the other variables did not.

Eight (16\%) patients displayed disease progression during the period of follow-up. They included three $(8 \%)$ of the $36 \mathrm{pTa}$ and five $(33 \%)$ of the 15 pT1 tumors and this difference was significant $(P=0.04)$. In a univariate analysis, high tumor stage was significantly associated with an increased risk of disease progression in our series of patients $(P=0.013$ by log-rank test) (Figure 3a). Disease progression also occurred in none $(0 \%)$ of the 13 G1, in five (17\%) of the $29 \mathrm{G} 2$ and in $3(33 \%)$ of the nine G3 tumors. These differences were significant (0.028 at $\chi^{2}$ test for trend) and in a univariate analysis a low tumor grade (G1) was significantly
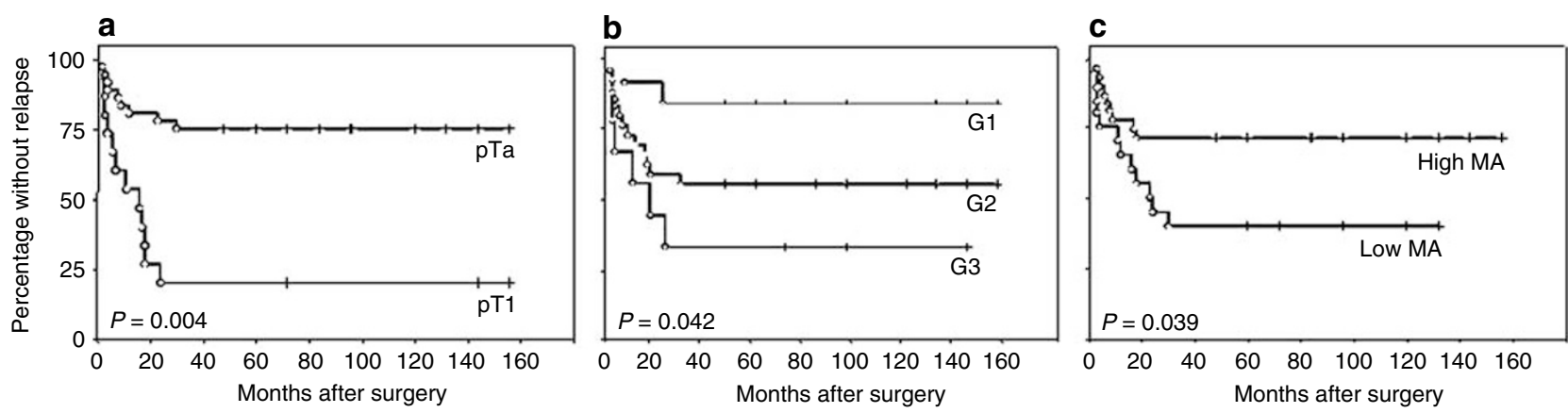

\begin{tabular}{lccc}
\hline & & \multicolumn{2}{c}{ Events } \\
& & observed (\%) & censored (\%) \\
\hline pTa & 36 & $9(25)$ & $27(75)$ \\
pT1 & 15 & $12(80)$ & $3(20)$ \\
\hline Total & 51 & $21(41)$ & $30(59)$
\end{tabular}

\begin{tabular}{|c|c|c|c|}
\hline & \multicolumn{3}{|c|}{ Events } \\
\hline & & observed (\%) & censored (\%) \\
\hline G1 & 13 & $2(15)$ & $11(85)$ \\
\hline G2 & 29 & $13(45)$ & $16(55)$ \\
\hline G3 & 9 & $6(67)$ & $3(33)$ \\
\hline Total & 51 & $21(41)$ & $30(59)$ \\
\hline
\end{tabular}

\begin{tabular}{lccc}
\hline & \multicolumn{3}{c}{ Events } \\
& & observed (\%) & censored (\%) \\
\hline High MA & 31 & $9(29)$ & $22(71)$ \\
Low MA & 20 & $12(60)$ & $8(40)$ \\
\hline Total & 51 & $21(41)$ & $30(59)$
\end{tabular}

Figure 2 Kaplan-Meier curves for disease-free survival in a series of 51 young ( $<45$ years) patients with superficial bladder cancer Patients were stratified by: (a) tumor stage; (b) tumor grade and (c) the frequency of microsatellite alterations (MA) (see the text for details). 

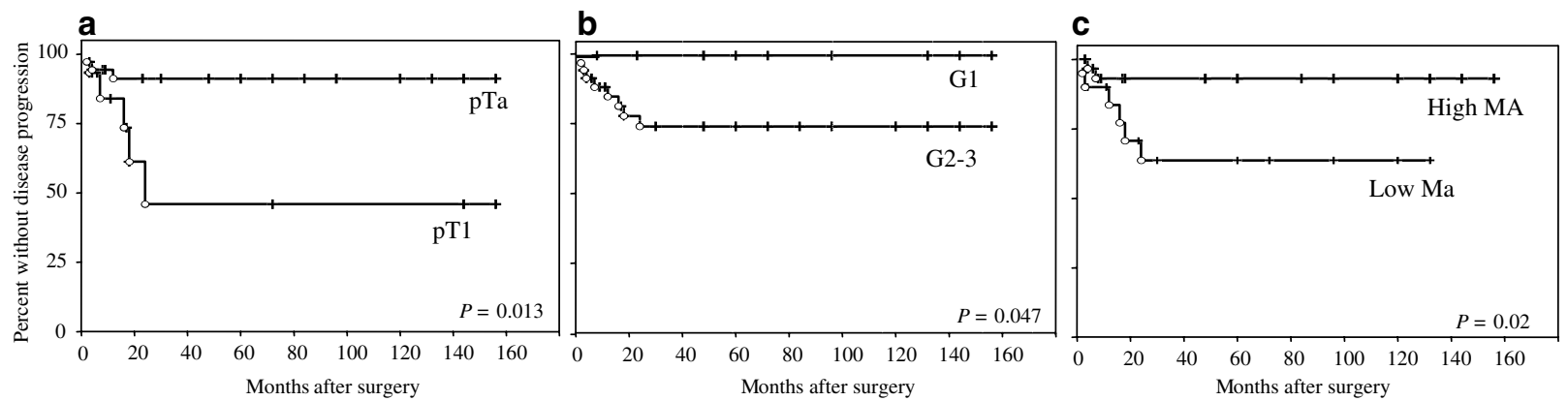

\begin{tabular}{lccc}
\hline & & \multicolumn{2}{c}{ Events } \\
& & observed (\%) & censored (\%) \\
\hline pTa & 36 & $3(8)$ & $33(92)$ \\
pT1 & 15 & $5(33)$ & $10(67)$ \\
\hline Total & 51 & $8(16)$ & $43(84)$
\end{tabular}

\begin{tabular}{lccc}
\hline & & \multicolumn{2}{c}{ Events } \\
& & observed (\%) & censored (\%) \\
\hline G1 & 13 & $0(0)$ & $13(100)$ \\
G2-3 & 38 & $8(21)$ & $30(79)$ \\
\hline Total & 51 & $8(16)$ & $43(84)$
\end{tabular}

\begin{tabular}{lccc}
\hline & & \multicolumn{2}{c}{ Events } \\
& & observed $(\%)$ & censored (\%) \\
\hline High MA & 31 & $2(6)$ & $29(94)$ \\
Low MA & 20 & $6(30)$ & $14(70)$ \\
\hline Total & 51 & $8(16)$ & $43(84)$
\end{tabular}

Figure 3 Kaplan-Meier curves for disease progression in a series of 51 young ( $<45$ years) patients with superficial bladder cancer. Patients were stratified by: (a) tumor stage; (b) tumor grade and (c) the frequency of microsatellite alterations (MA) (see the text for details). Analysis could not be performed by stratifying patients according to each tumor grade (G1, G2 and G3) since no G1 tumors showed disease progression during the period of follow-up.

associated with a reduced risk of disease progression ( $P=0.047$ by log-rank test) in our series of patients (Figure $3 \mathrm{~b}$ ).

Two $(6 \%)$ of the 31 cases displaying high frequency of microsatellite alterations and six $(30 \%)$ of the remaining ones showed disease progression in our series of bladder urothelial carcinomas during the period of follow-up and this difference was significant $(P=0.04)$ (Table 3$)$. The Kaplan-Meier curves displayed a significant separation when tumors with high frequency of microsatellite alterations were stratified vs the remaining ones demonstrating that the occurrence of alterations at the selected loci was associated with a reduced risk of disease progression in a univariate analysis $(P=0.022$ by log-rank test $)$ (Figure 3c).

In a multivariate analysis performed by building a Cox proportional hazards model that included tumor stage, tumor grade and frequency of microsatellite alterations, only higher tumor stage $(P=0.03$; relative risk $=1.9 ; 95 \%$ confidence interval: 1.01-25.01) confirmed to be an independent predictor of disease progression in our series of patients while the presence of a low frequency of microsatellite alterations remained not far from significance $(P=0.05)$ with a relative risk of 1.67 (95\% confidence interval: 0.994-32.05).

\section{Microsatellite Alteration at Each Locus}

The microsatellite that was most frequently altered in our series of bladder urothelial carcinomas was D11S488 (69\%), followed by D9S162 (61\%), D3S3050 (55\%), D3S1300 (51\%) and D4S243 (SHGC) (51\%). All the other loci analyzed were altered in less than $50 \%$ of cases (Table 2 ).
The relationship between the occurrence of alterations at each locus and other clinicopathological parameters is shown in Table 4. No relationship was observed between alterations in any of the loci analyzed and patients gender (data not shown). None of the loci showed a different frequency of alterations between pTa and pT1 tumors. As far as tumor grade is concerned, a significant trend for a progressive reduction in the frequency of alterations with increasing tumor grade was observed for the microsatellite locus D9S164 $(P=0.02)$. No other association between tumor grade and alterations at specific loci was observed.

We could not detect a significant relationship between alterations at a specific microsatellite and tumor recurrence/progression for any of the loci analyzed. However, the Kaplan-Meier curves of disease-free survival showed that the occurrence of microsatellite alterations at the D5S346 locus was associated with a reduced risk of recurrence in a univariate analysis in our series of young patients ( $P=0.039$ by log-rank test) (data not shown). A significant separation was also observed for the Kaplan-Meier curves of disease progression when patients were stratified according to the alterations in the same locus, but the difference was not significant ( $P=0.05$ by log-rank test) (data not shown).

\section{Discussion}

In this study, a series of 19 microsatellite loci, previously reported to be associated with bladder tumorigenesis, were analyzed to detect microsatellite alterations in a subset of superficial (pTa, pT1) bladder urothelial carcinomas arising at young age. We found that microsatellite alterations, in the form of deletion ( $\mathrm{LOH})$ or modification of 
Table 4 Microsatellite alterations at 19 loci in a series of 51 superficial papillary bladder urothelial carcinomas arising in young patients in relationship with clinicopathological parameters

\begin{tabular}{|c|c|c|c|c|c|c|c|c|}
\hline \multirow[t]{2}{*}{ Locus } & \multicolumn{8}{|c|}{ Number of alterations $(\%)^{\mathrm{a}}$} \\
\hline & Total & $p T a$ & $p T 1$ & G1 & G2 & G3 & Non-recur. ${ }^{\mathrm{b}}$ & Recur. \\
\hline D1S2883 & $24(47)$ & $15(62)$ & $9(38)$ & $6(25)$ & $14(58)$ & $4(17)$ & $15(62)$ & $9(38)$ \\
\hline D3S3050 & $28(55)$ & $19(68)$ & $9(32)$ & $6(21)$ & $19(68)$ & $3(11)$ & $17(61)$ & $11(39)$ \\
\hline D3S1300 & $26(51)$ & $19(73)$ & $7(27)$ & $7(27)$ & $15(58)$ & $4(15)$ & $16(62)$ & $10(38)$ \\
\hline D3S2418 & $15(29)$ & $11(73)$ & $4(27)$ & $4(27)$ & $9(60)$ & $2(13)$ & $11(73)$ & $4(27)$ \\
\hline D4S243-(SHGC) & $26(51)$ & $18(69)$ & $8(31)$ & $7(27)$ & $16(61)$ & $3(12)$ & $18(69)$ & $8(31)$ \\
\hline D4S243-(MH34) & $25(49)$ & $17(68)$ & $8(32)$ & $6(24)$ & $15(60)$ & $4(16)$ & $17(68)$ & $8(32)$ \\
\hline D5S346 & $20(39)$ & $13(65)$ & $7(35)$ & $4(20)$ & $12(60)$ & $4(20)$ & $15(75)$ & $5(25)$ \\
\hline D9S54 & $14(27)$ & $11(79)$ & $3(21)$ & $3(21)$ & $8(58)$ & $3(21)$ & $11(79)$ & $3(21)$ \\
\hline D94S747 & $18(35)$ & $12(67)$ & $6(33)$ & 3 (17) & $13(72)$ & $2(11)$ & $8(44)$ & $10(56)$ \\
\hline D9S162 & $31(61)$ & $23(74)$ & $8(26)$ & $8(26)$ & $20(64)$ & $3(10)$ & $21(68)$ & $10(32)$ \\
\hline IFNA & $6(12)$ & $4(67)$ & $2(33)$ & $1(17)$ & $4(66)$ & $1(17)$ & $3(50)$ & $3(50)$ \\
\hline D9S171 & $24(47)$ & $20(83)$ & $4(17)$ & $6(25)$ & $16(67)$ & $2(8)$ & $14(58)$ & $10(42)$ \\
\hline D9S164 & $22(43)$ & $17(77)$ & $5(23)$ & $8(36)$ & $13(59)$ & $1(5)$ & $15(68)$ & $7(32)$ \\
\hline D11S488 & $35(69)$ & $26(74)$ & $9(26)$ & $9(26)$ & $22(63)$ & $4(11)$ & $22(63)$ & $13(37)$ \\
\hline D13S802 & $25(49)$ & $16(64)$ & $9(36)$ & $7(28)$ & $11(44)$ & $7(28)$ & $13(52)$ & $12(48)$ \\
\hline D16S310 & $23(45)$ & $14(61)$ & $9(39)$ & $3(13)$ & $19(83)$ & $1(4)$ & $13(57)$ & $10(43)$ \\
\hline D16S476 & $15(29)$ & $9(60)$ & $6(40)$ & $3(20)$ & $11(73)$ & $1(7)$ & $10(67)$ & $5(33)$ \\
\hline D17S654 & $16(31)$ & $13(81)$ & 3 (19) & $5(31)$ & $9(56)$ & $2(13)$ & $11(69)$ & $5(31)$ \\
\hline TP53 & $13(25)$ & $8(61)$ & 5 (39) & $4(31)$ & $6(46)$ & $3(23)$ & $7(54)$ & $6(46)$ \\
\hline
\end{tabular}

${ }^{\mathrm{a}}$ The values indicate the number of alterations identified for each locus in our series of tumors keeping in account both shifts in the DNA peak and deletions.

$\mathrm{b}_{\text {recur. }}=$ recurrent

repetitive sequences, were a frequent event in young bladder tumorigenesis and were detectable at one or more loci in all of the cancers analyzed (Tables 2-4). A surprising result of this study was that the occurrence of microsatellite alterations was more frequent in low-grade and low-stage tumors (Table 2), although the difference did not reach statistical significance, and in a univariate analysis was predictor of a longer disease-free survival and a reduced risk of disease progression (Figures 2c and 3c).

Frequent occurrence of loss of microsatellites has been described in bladder urothelial carcinomas ${ }^{5}$ and $\mathrm{LOH}$ has been reported to occur on several chromosomes $^{5-7,8}$ in both superficial (pTa-T1) and muscle-invasive, but no data are available on its frequency in bladder cancers arising at young age. As far as microsatellite instability is concerned, conflicting data have been reported in the literature about bladder cancers with mutation frequencies ranging from 0 to $100 \% .{ }^{10-17}$ These discrepancies, that do not allow to draw any definitive conclusion on the frequency of microsatellite instability in bladder cancer, can be attributed to different factors, such as the source of tumor DNAs used for the analysis, the sample size, the grade and stage of tumors analyzed and the numbers and types of microsatellites included in each study. To override these problems, we used laser microdissection that allows the preparation of high-quality microdissected tissues as source of DNA suitable for subsequent molecular analysis. This is very important since the contamination due to normal cells is a potentially relevant confusing factor and can compromise the ability of detecting microsatel- lite alterations using PCR-based assays. We also choose to focus on a selected subset of patients and, to our knowledge, this is the first study analyzing a large number of microsatellite loci on a homogeneous (only superficial cases) group of papillary bladder cancers arising at a young age.

Microsatellite instability is primarily a feature of HNPCC-related tumors but it also occurs in a subset of most sporadic human cancers. ${ }^{27}$ Conflicting results have been reported on the frequency of microsatellite instability in many tumors, including bladder cancers, and differences in the microsatellites analyzed have, in part, contributed to these discrepancies. Thus, to standardize detection a panel of repeat loci (the Bethesda consensus panel) was established to define microsatellite instability. ${ }^{26}$ The panel only included mono- and dinucleotide repeats that are mainly affected by DNA mismatch repair deficiency. A second variety of instability has been more recently reported that appears to be not related to DNA mismatch repair deficiency and is best seen at tetranucleotide repeats. ${ }^{11}$ This form of instability has been termed elevated microsatellite alterations at selected tetranucleotides and appears to occur with high frequency (approximately 40\%) in bladder cancer. ${ }^{11,18}$

We preferred to not use the Bethesda consensus panel of microsatellites since bladder urothelial carcinoma is not found at a higher incidence in HNPCC kindred ${ }^{27}$ and no relationship has been observed between the expression of DNA mismatch repair proteins and the occurrence of microsatellite instability in bladder cancer. ${ }^{28}$ Thus, we selected a series of 19 loci that have been previously reported 
to be altered in bladder tumorigenesis and choose to not include mononucleotides but only dinucleotide and larger repeat sequences, including tetranucleotides, since they tend to be more frequently altered in bladder cancer. ${ }^{6,7,11,18}$

We found that the occurrence of microsatellite alterations was a frequent and early event in young bladder tumorigenesis and was detectable, at one or more loci, in all of the cancers analyzed independently of tumor grade and stage. This finding is in agreement with the only previous study in which different microsatellites were analyzed in 14 bladder tumors arising at young age (35-55 years) and alterations were detected in several microsatellite loci in all tumors. ${ }^{17}$ The mechanisms responsible for microsatellite alterations are still not completely understood. As previously mentioned, microsatellite instability is mainly found in a subset of hereditary cancers and is associated with germline mutations in genes involved in DNA repair mechanisms. ${ }^{9}$ It has been hypothesized that bladder cancers arising at young age might also be hereditary. ${ }^{9}$ Our data do not allow to draw any conclusion from this point of view. However, the type of alterations observed coupled with the high occurrence of $\mathrm{LOH}$ seems to support a distinctive genotype compared to DNA mismatch repair deficiency. On the other hand, microsatellite alterations might represent an acquired genetic alteration that arises during the process of malignant transformation of bladder urothelium and the high frequency of alterations in tetranucleotide repeats (detected in all but two of the tumors included in this study) support the hypothesis of a major role played by elevated microsatellite alterations at selected tetranucleotides in young bladder tumorigenesis as already reported for the older counterpart. ${ }^{11,18}$ We are currently analyzing alterations in the same set of loci in a series of bladder urothelial carcinomas arisen at an older age and preliminary data suggest a reduced occurrence of alterations compared to young patients (MM and AS, preliminary observations) thus supporting the hypothesis of a higher incidence of microsatellite alterations in young bladder tumorigenesis.

The occurrence of microsatellite alterations is expression of a loss of DNA repair efficiency. It is possible that this deficiency contribute to carcinogenesis by increasing the mutation rate of genes directly involved in cell transformation (ie, oncogenes and tumor suppressor genes) or that, on the other hand, it is caused by mutations in specific genes. Cell cycle deregulation has been reported to play a role in genetic instability observed in tumor cells. ${ }^{21}$ Indeed, the occurrence of elevated microsatellite alterations at selected tetranucleotides was reported to relate to p53 mutations in superficial bladder cancer. ${ }^{18}$ Consistent with our results, however, this association was not evident with p53 immunostaining that can give a positive staining also in the absence of p53 gene mutation. ${ }^{18}$ We also did not observe any significant relationship with the expression levels of cyclin D1 and of the CDK inhibitor p27 (Table 3). A significant association, however, was observed between the occurrence of microsatellite alterations and the proliferation index, as assessed by the proliferation marker MIB-1, in our series of tumors (Table 3). The significance of this finding remains to be defined.

Regardless of the underlying molecular mechanisms, determining whether particular cancers show microsatellite alterations may have important biological and clinical implications. In fact, identification of microsatellite alterations can help understanding tumor pathogenesis, might provide a diagnostic tool to detect rare cancer cells in clinical samples and, finally, it might also have prognostic importance. In this study, we identified a set of five markers (D11S488, D9S162, D3S3050, D3S1300 and D4S243) that are altered at early stages of young bladder tumorigenesis and have the potentiality to allow detection of $100 \%$ of bladder cancers since at least one of them was found altered in all tumors (see Tables 2 and 4 and data not shown). This finding is of great interest in view of the possibility to use PCR-based microsatellite analysis of the exfoliated cells, alone or in combination with cytology, for screening primary and recurrent bladder cancer cells in urine sediments. ${ }^{10,11}$ If the results of this study will be confirmed using cytological urine specimens, analysis of these loci might have a great clinical potential as reliable, noninvasive method to detect malignant cells in the urinary sediment for both follow-up of young bladder cancer patients and screening of high-risk populations. This is of great importance since a reliable assay on urine may greatly benefit the patients and help clinicians to timely identify recurrences and select the most appropriate treatment, especially in cases, that is, after radiotherapy, when interpretation of cytology, as well as cystoscopy, is often problematic.

In conclusion, we demonstrated that microsatellite alterations, in the form of instability or deletion, are a frequent and early event in bladder cancers arising at young age suggesting a possible role in the process of tumor development. Moreover, it might have a prognostic significance in this subset of patients being a predictor of favorable prognosis, if the results of the present study will be confirmed on a larger cohort of patients. Finally, the high frequency of alterations at an early stage of disease support the suitability of microsatellite analysis for early detection of cancer cells in urine sediment for diagnosis and/or follow-up of bladder cancers in young patients.

\section{Acknowledgements}

This work was supported in part by grants from Università Cattolica and MIUR (Programma 
Oncologia L 449/97) (to AC), and the Associazione Italiana per la Ricerca sul Cancro (to AS).

\section{References}

1 Parkin DM. Global cancer statistics in the year 2000. Lancet Oncol 2001;2:533-543.

2 Kutarski PW, Padwell A. Transitional cell carcinoma of the bladder in young adults. Br J Urol 1993;72:749-755.

3 Hearn CM, Ghosh S, Todd JA. Microsatellites for linkage analysis of genetic traits. Trends Genet 1992;8: 288-294.

4 Weissenbach J, Giyapay G, Dib C, et al. A second generation linkage map of the human genome. Nature (London) 1992;359:794-801.

5 McNeil C. DNA test for bladder cancer hits a high mark. J Natl Cancer Inst 1996;88:146-147.

6 Chang W, Cairns P, Schoenberg M, et al. Novel suppressor loci on chromosome $14 \mathrm{q}$ in primary bladder cancer. Cancer Res 1995;55:3246-3249.

7 Smeets W, Pauwels R, Laarakkers L, et al. Chromosomal analysis of bladder cancer: nonrandom alteration. Cancer Genet Cytogen 1987;29:29-41.

8 Miyao N, Tsai Y, Lerner S, et al. Role of chromosome 9 in human bladder cancer. Cancer Res 1993;53: 4066-4070.

9 Loeb LA. Microsatellite instability: marker of a mutator phenotype in cancer. Cancer Res 1994;54: 5059-5063.

10 Mao L, Schoenberg MP, Scicchitano M. Molecular detection of primary bladder cancer by microsatellite analysis. Science 1996;271:659-662.

11 Mao L, Lee DJ, Tockman MS, et al. Microsatellite alterations as clonal markers for the detection of human cancer. Proc Natl Acad Sci USA 1994;91: 9871-9875.

$12 \mathrm{Li}$ M, Zhang ZF, Reuter VE, et al. Chromosome 3 allelic losses and microsatellite alterations in transitional cell carcinoma of the urinary bladder. Am J Pathol 1996; 149:229-235.

13 Uchida T, Wang C, Wada C, et al. Microsatellite instability in transitional cell carcinoma of the urinary tract and its relationship to clinicopathological variables and smoking. Int J Cancer 1996;69: 142-145.

14 Linnenbach AJ, Robbins SL, Seng BA, et al. Urothelial carcinogenesis. Nature 1994;367:419-420.

15 Gonzales-Zulueta M, Ruppert JM, Tokini K, et al. Microsatellite instability in bladder cancer. Cancer Res 1993;53:5620-5623.
16 Bonnal C, Ravery V, Toublanc M, et al. Absence of microsatellite instability in transitional cell carcinoma of the bladder. Urology 2000;55:287-291.

17 Christensen M, Jensen MA, Wolf H, et al. Pronounced microsatellite instability in transitional cell carcinomas from young patients with bladder cancer. Int J Cancer (Pred Oncol) 1998;79:396-401.

18 Danaee H, Nelson H, Karagas M, et al. Microsatellite instability at tetranucleotide repeats in skin and bladder cancer. Oncogene 2002;21:4894-4899.

19 Migaldi M, Sgambato A, Garagnani L, et al. Loss of p21 Waf1 expression is a strong predictor of reduced survival in primary superficial bladder cancers. Clinical Cancer Res 2000;6:3131-3138.

20 Sgambato A, Migaldi M, Faraglia B, et al. Cyclin D1 expression in papillary superficial bladder cancer: Its association with other cell cycle-associated proteins, cell proliferation and clinical outcome. Int J Cancer 2002;97:671-678.

21 Sgambato A, Flamini G, Cittadini A, et al. Abnormalities in cell cycle control in cancer and their clinical implications. Tumori 1998;84:421-433.

22 Fleshner NE, Herr HW, Stewart AK, et al. The National Cancer Data Base report on bladder carcinoma. The American College of Surgeons Commission on Cancer and the American Cancer Society. Cancer 1996;7: 1505-1513.

23 Mostofi FH, Sobin LH, Torloni H. Histological typing of urinary bladder tumours. In: Springer (ed). International Classification of Tumours, 10 edn. World Health Organisation: Geneva, 1973.

24 Page D, Fleming I, Fritz A, et al. Urinary bladder. In: Greene F (ed). AJCC Cancer Staging Manual, 6th edn. Springer-Verlag: New York, 2002.

25 Sgambato A, Migaldi M, Faraglia B, et al. Loss of $\mathrm{p} 27^{\mathrm{Kip} 1}$ expression correlates with tumor grade and with reduced disease-free survival in primary superficial bladder cancers. Cancer Res 1999;13: 3245-3250.

26 Boland C, Thibodeau S, Hamilton S, et al. A National Cancer Institute Workshop on Microsatellite Instability for cancer detection and familial predisposition: development of international criteria for the determination of microsatellite instability in colorectal cancer. Cancer Res 1998;58:5248-5257.

27 Arzimanoglou I, Gilber F, Barber H. Microsatellity instability in human solid tumors. Cancer 1998;82: 1802-1820.

28 Catto J, Xinarianos G, Burton J, et al. Differential expression of hMLH1 and hMSH2 is related to bladder cancer grade, stage and prognosis but not microsatellite instability. Int J Cancer 2003;105: $484-490$. 